\title{
Original
}

\section{Assessment of the Bone Regenerative Process from Fibular Periosteum by in vivo Micro Computed Tomography}

\author{
Takayuki Mashimo ${ }^{1,2)}$, Tadahito Saito ${ }^{1,2)}$, Hiroshi Shiratsuchi ${ }^{1,2)}$, Jun Iwata ${ }^{1,2)}$, Takeshi Uryu ${ }^{1,2)}$, \\ Takaaki Tamagawa',2), Shunsuke Namaki'), Kunihito Matsumoto ${ }^{3)}$, Shouji Kawashima ${ }^{3)}$, \\ Yoshiyuki Mori' ${ }^{4}$, Yoshinori Arai ${ }^{5)}$, Kazuya Honda ${ }^{3,6)}$ and Yoshiyuki Yonehara ${ }^{2,7)}$ \\ 1) Division of Oral Structural and Functional Biology, Nihon University Graduate School of Dentistry, Tokyo, Japan \\ 2) Department of Oral and Maxillofacial Surgery, Nihon University School of Dentistry, Tokyo, Japan \\ 3) Department of Oral and Maxillofacial Radiology, Nihon University School of Dentistry, Tokyo, Japan \\ 4) Department of Oral and Maxillofacial Surgery, The University of Tokyo Hospital, Tokyo, Japan \\ 5) Nihon University School of Dentistry, Tokyo, Japan \\ 6) Division of Advanced Dental Treatment, Dental Research Center, Nihon University School of Dentistry, Tokyo, Japan \\ 7) Division of Systemic Biology and Oncology, Dental Research Center, Nihon University School of Dentistry, Tokyo, Japan \\ (Accepted for publication, June 20, 2013)
}

\begin{abstract}
The aim of this study was to examine the bone regenerative process from fibular periosteum in rats. Twenty male Wistar rats were divided into two groups : a periosteum preservation (PP) group $(\mathrm{n}=15)$ and a periosteum removal (PR) group. In the PP group, the fibula was totally removed, but the periosteum and blood supply were preserved. In the PR group $(n=5)$, the fibula was totally removed, including the periosteum. Radiological and histological findings were evaluated after operation. In the PP group, the increase in regenerative bone volume was highest at 1 week. At 2 weeks, the bone volume decreased transiently, but then continued to increase gradually until 4 weeks. There was little change after 4 weeks. The regenerative bone mineral density continued to increase gradually from 5 days until 8 weeks. In the PR group, there was no evidence of regenerative bone. These results suggest that the periosteum has osteogenic capacity and the peak of bone regeneration from the periosteum occurs around 4 to 6 weeks.
\end{abstract}

Key words: Fibula, Periosteum, Regenerative bone, Micro CT

\section{Introduction}

Maxillofacial bone defects resulting from tooth extraction, trauma, or tumor resection have been repaired with autogenous bone grafts or artificial materials. Autogenous bone grafting is generally an effective technique, but has disadvantages such as donor-site morbidity. Artificial materials have been developed to overcome the disadvantages of autogenous bone grafts, but can cause adverse effects such as foreign-body reactions. Since both autogenous bone grafts and artificial materials have inherent advantages and disadvantages, it is necessary to develop new reconstruction materials. We have focused on the use of periosteum as a new material with low morbidity and no foreign-body reactions. The use of bone regenerated from periosteal grafts is considered clinically significant.

The periosteum is a connective tissue that covers the surface

Correspondence to: Dr. Takayuki Mashimo, Department of Oral and Maxillofacial Surgery, Nihon University School of Dentistry, 1-8-13 Kanda-Surugadai, Chiyoda-ku, Tokyo 101-8310, Japan; Tel: +81-3-32198102; Fax:+81-3-3219-8355; E-mail: deta10027@g.nihon-u.ac.jp of bone. This tissue consists of two layers, a thick outer fibrous layer and an inner osteogenic layer (cambium layer) ${ }^{1)}$. The cambium layer plays an important role in osteogenesis. The osteogenic capacity of periosteum was first reported by Duhamel et al. ${ }^{2)}$ in 1739. However, stable results with free periosteum grafts were not obtained in experimental studies, and the osteogenic potential of periosteum remained controversial ${ }^{3-5}$. Clinically, Skoog ${ }^{6,7)}$ introduced the use of maxillary periosteal flaps for primary repair of alveolar clefts in the 1960s. Ritisila et al. ${ }^{8)}$ used free periosteal grafts from the tibia for primary repair of the palate. These reports restimulated interest in the osteogenic capacity of periosteum. Several studies reported that vascularized periosteum has good osteogenic properties, suggesting that vascularity has an important role in periosteum-related osteogenesis ${ }^{9-12}$. In addition, Acland and van den Wildenberg et al. ${ }^{13,14)}$ reported that mechanical stress influences the osteogenic capacity of periosteum. Takato et al. ${ }^{15,16)}$ found that the osteogenic capacity of periosteum depends not only on weight and stress, but also. on the volume of periosteum and the blood circulation. Uddströmer et al. ${ }^{17,18)}$ 

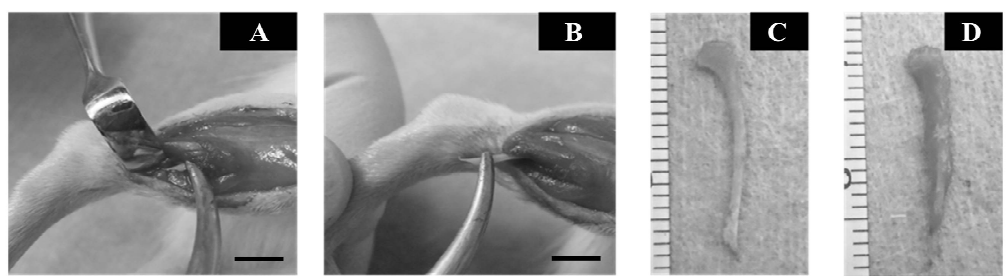

Figure 1. Intraoperative images.

A: The epiphysis of the fibula was held by surgical clips. (scale bar: $5 \mathrm{~mm}$ ); B: The fibula was pulled out in the peripheral direction with the surgical clips. (scale bar: $5 \mathrm{~mm}$ ); C: The removed fibula with the periosteum preserved in the PP model. (18 $\mathrm{mm}$ in length); D: The removed fibula with the periosteum removed in the PR model. (18 $\mathrm{mm}$ in length)
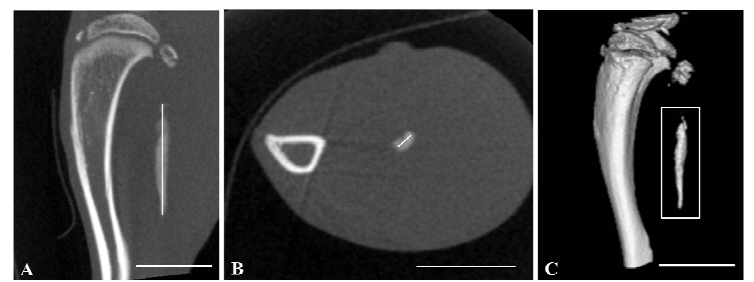

Figure 2. Measurement of regenerative bone.

A: The line indicates the longest length of regenerative bone in the longitudinal plane images. (scale bar: $5 \mathrm{~mm}$ ); B: The line indicates the longest diameter of regenerative bone in the cross-sectional plane images. (scale bar: $5 \mathrm{~mm}$ ); $\mathrm{C}$ : The square indicates the measuring range of regenerative bone volume and mineral density. (scale bar: 5 $\mathrm{mm})$

demonstrated that periosteum is intimately involved in fracture healing. Oni et al. ${ }^{19,20)}$ found that removing the periosteum prevents fracture healing. Li et al. ${ }^{21)}$ showed that cartilaginous and osteoid bone derived from periosteum participate in fracture healing. Thus, the periosteum has been reported to have osteogenic potential, but the bone regenerative process from periosteum, including the time course of osteogenesis, regenerative bone volume and bone mineral density, remains poorly understood.

Vascularized fibula grafting has been shown to be an effective technique for reconstruction of the maxillofacial region ${ }^{22,23)}$. However, the disadvantages of this technique are restricted availability of suitable grafts and high invasion associated with acquiring bone from donor sites. If regenerative bone from fibular periosteum could be used in place of direct bone grafts, it would be possible to reconstruct jaw bones without directly using the fibula.

A better understanding of the timing and properties of regenerative bone from periosteum may provide important clues to the optimal timing of periosteal grafting. Recently, most studies of periosteum examined the repair process in segmental bone defect models and bone fracture models ${ }^{24-27)}$. Few studies have focused exclusively on fibular periosteum. Studies examining the details of bone regeneration from periosteum in the same subjects are extremely rare.

A recently developed micro-computed tomography (microCT) system, R_mCT ${ }^{\circledR}$ (Rigaku Co., Tokyo, Japan), has made it possible to obtain images of anesthetized experimental animals. Clear hard tissue images of small animals can be obtained with short exposure time and low dose. A lot of studies have proven the value of micro-CT for the assessment of changes over time in the animal experiments ${ }^{28,29)}$. Saito et al. ${ }^{30)}$ have concluded that micro-CT is suitable for evaluating bone regenerative process after mandibular condylectomy in rat model and periosteum plays a critical role in bone regeneration of mandibular condyle. However, the detail of regenerative process of long bones such as fibula is unclear. Thus, we conducted the assessment of the detailed process of bone regeneration from fibular periosteum of experimental animals over time using micro $\mathrm{CT}$.

\section{Materials and Methods}

\section{Animals}

Twenty 6-week-old male Wistar rats with a mean body weight of $130 \mathrm{~g}$ (Sankyo Laboratory, Tokyo, Japan) were used in the study. The rats were divided into two groups : a periosteum preservation $(\mathrm{PP})$ group $(\mathrm{n}=15)$ and a periosteum removal $(\mathrm{PR})$ group $(\mathrm{n}=5)$. The animals were housed in an experimental animal room $\left(22^{\circ} \mathrm{C}\right.$, $55 \%$ relative humidity, and a 12 -h light/dark cycle) and fed a standard laboratory diet. Water was provided ad libitum. The Animal Experimentation Committee of the Nihon University School of Dentistry approved this study.

\section{Surgical Procedure}

All surgical procedures were performed with the animals under intraperitoneal anesthesia with sodium pentobarbital $(50 \mathrm{mg} / \mathrm{kg}$ body weight; Somnopentyl, Schering-Plough, Munich, Germany). The surgical site was shaved, and the skin was washed with $70 \%$ ethanol. The site was then locally anesthetized with an intramuscular injection of $1 \mathrm{ml} 2 \%$ lidocaine (Xylocaine, AstraZeneca, Osaka, Japan). A lateral skin incision about $20 \mathrm{~mm}$ in length was made in the lower leg, and a smaller incision was made into the gastrocnemius muscle. The muscle was retracted to expose the connections of the tibia and fibula. An osteotomy was performed at the epiphysis of the fibula.

In the PP group, the gastrocnemius muscle and periosteum were detached from the fibular diaphysis, and the fibula was totally 
Takayuki Mashimo et al. Bone Regenerative Process from Periosteum

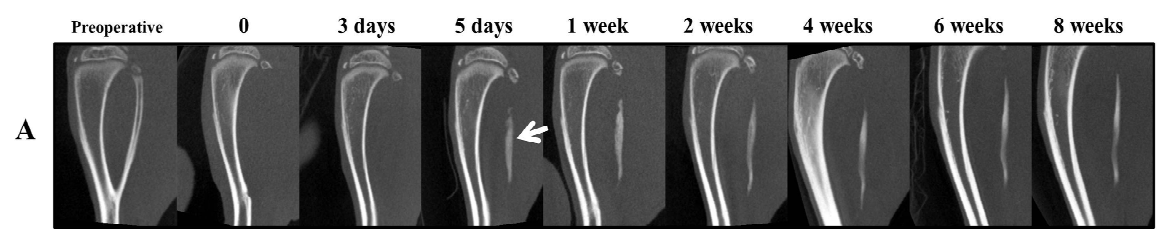

B
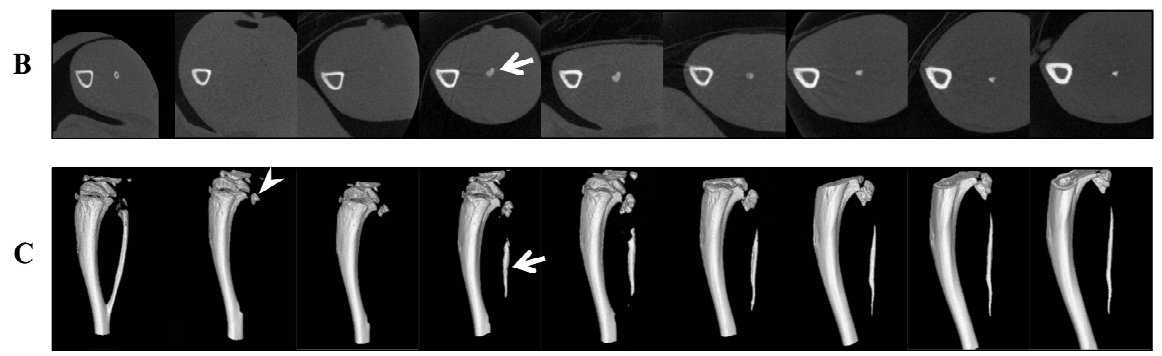

Figure 3. CT images in the PP model.

A: Longitudinal plane images are shown. The yellow arrow indicates the regenerative bone; B: Cross-sectional plane images are shown. The indicates the regenerative bone; $\mathrm{C}$ : Three-dimensional (3D) images are shown. The arrow indicates the regenerative bone, and the yellow arrowhead indicates the articular cartilage.
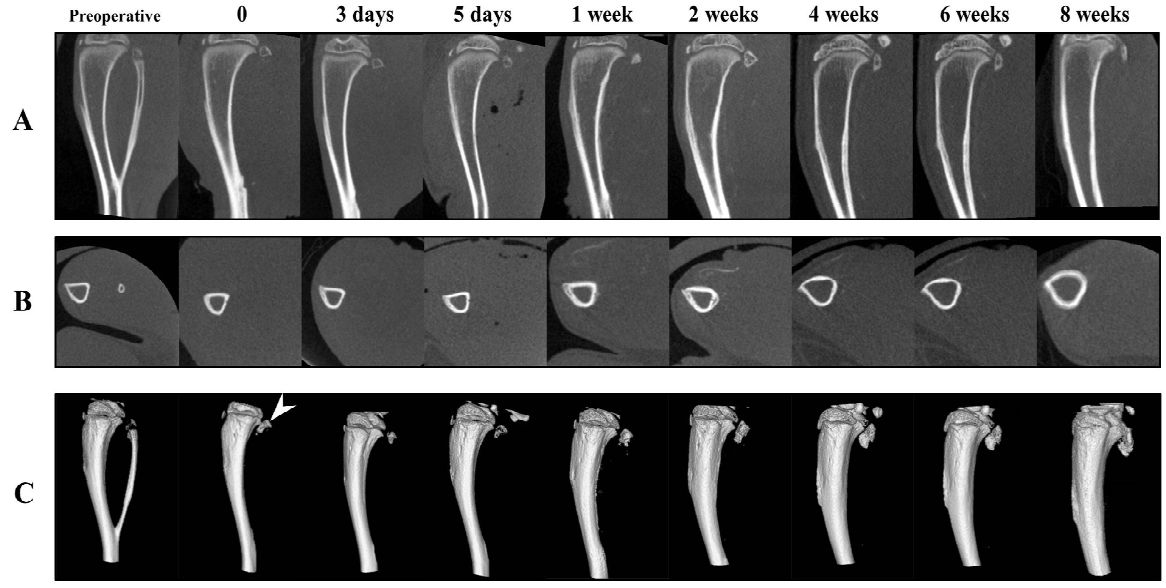

Figure 4. CT images of the PR model.

A: Longitudinal plane images are shown. No regenerative bone was observed; B: Cross-sectional plane images are shown. No regenerative bone was observed; C: Three-dimensional (3D) images are shown. No regenerative bone was observed, but the articular cartilage was evident ( arrowheads).

removed by pulling peripherally with the use of surgical clips; the blood supply to the periosteum was maintained (Fig. 1-A, B, C). In the PR group, the same procedure was performed; however, the periosteum was not detached and removed with the fibula (Fig. 1-D). After the fibula was removed, the muscle and skin were closed with 5-0 nylon sutures (Bear Medic Co., Tokyo, Japan). Rats were sacrificed 5 at days and 1, 2, 4, 6, and 8 weeks after operation.

\section{Radiographic analysis}

Before operation, immediately after operation, and 3 days, 5 days, and 1, 2, 4, 6, and 8 weeks after operation, the bone-defect region underwent radiographic analysis by in vivo micro computed tomography system (R_mCT; Rigaku Co., Tokyo, Japan). Longitudinal, cross-sectional, and 3-dimensional images (3D images) were examined. During exposure, the rats were anesthetized with isoflurane (DS Pharma Animal Health Co. Ltd., Osaka, Japan). The exposure conditions were 17 seconds at 90 $\mathrm{kv} / 100 \mathrm{~mA}$. The image volume was a cylinder $2.4 \mathrm{~cm}$ in diameter and $2.4 \mathrm{~cm}$ high with a voxel matrix size set to $481 \times 481 \times 483$. The longest lengths and diameters of regenerative bone were measured in millimeters ( $\mathrm{mm}$ ) with the use of image reconstruction software (i-VIEW-R; Morita Co., Kyoto, Japan) (Fig. 2-A, B).

\section{Quantitative analysis}

The bone volume and bone mineral density of the whole regenerative bone were measured in cubic millimeters $\left(\mathrm{mm}^{3}\right)$ and milligrams per cubic centimeter $\left(\mathrm{mg} / \mathrm{cm}^{3}\right)$ with the use of quantitative analysis software (3-by-4 viewer 2011, Kitasenju Radist Dent, Tokyo, Japan) (Fig. 2-C). 


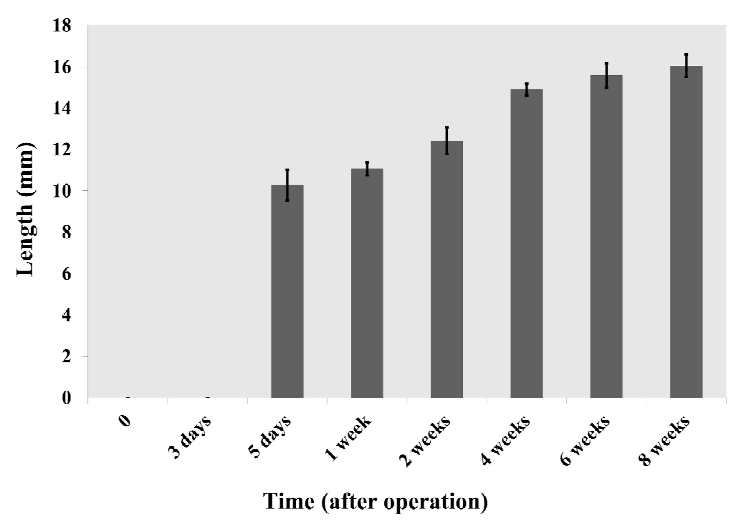

Figure 5. Time course of the length of the regenerative bone. The length tended to increase gradually until 8 weeks. Each bar indicates the mean \pm SD of five independent experiments.

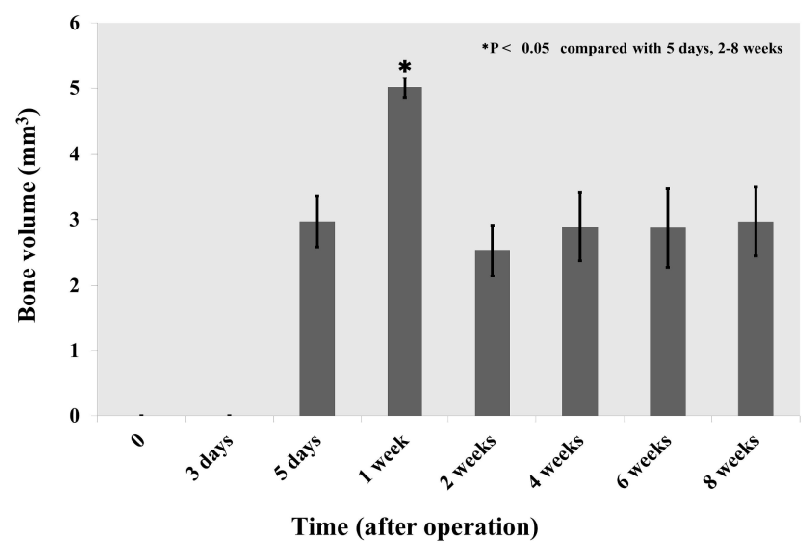

Figure 7. Time course of regenerative bone volume.

Bone volume increased remarkably at 1 week $(p<0.05)$, but decreased at 2 weeks. After 2 weeks, bone volume increased slightly up to 4 weeks. Little change was seen from 4 weeks to 8 weeks. Each bar indicates the mean $\pm \mathrm{SD}$ of five independent experiments.

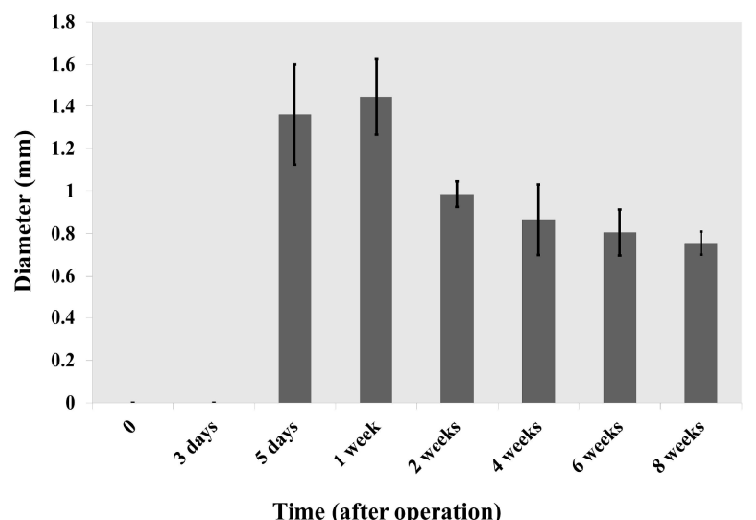

Figure 6. Time course of the diameter of the regenerative bone. The diameter of the cross-sectional image was thickest at 1 week, but then gradually decreased. Each bar indicates the mean \pm SD of five independent experiments.

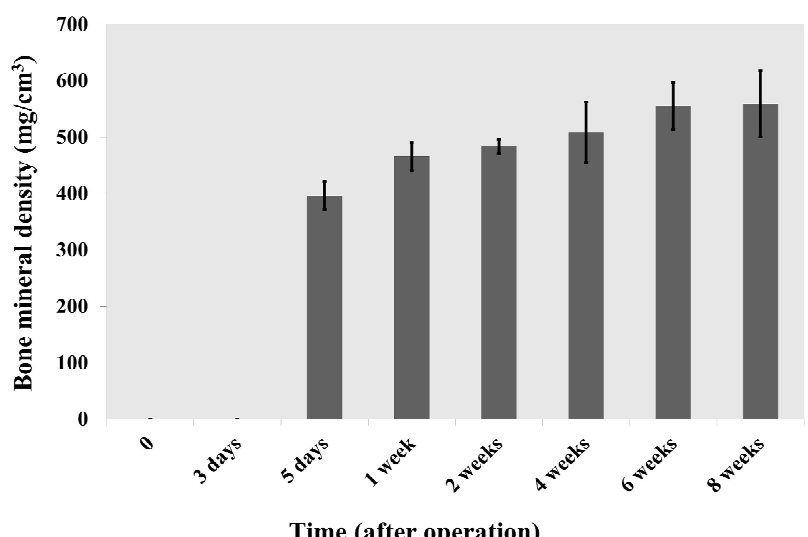

Figure 8. Time course of regenerative bone mineral density. Bone mineral density continued to increase gradually up to 6 weeks. There was little change between 6 and 8 weeks. Each bar indicates the mean \pm SD of five independent experiments.

Table 1. Mean of Each Measurements in the Regenerative Bone

\begin{tabular}{llllllll}
\hline & 3 days & 5 days & 1 week & 2 weeks & 4 weeks & 6 weeks & 8 weeks \\
\hline Length of bone $(\mathrm{mm})$ & 0 & $10.29(0.73)$ & $11.07(0.31)$ & $12.43(0.64)$ & $14.90(0.29)$ & $15.60(0.59)$ & $16.06(0.54)$ \\
Diameter of bone $(\mathrm{mm})$ & 0 & $1.36(0.24)$ & $1.45(0.18)$ & $0.99(0.06)$ & $0.87(0.17)$ & $0.81(0.11)$ & $0.76(0.05)$ \\
Bone volume $\left(\mathrm{mm}^{3}\right)$ & 0 & $2.97(0.39)$ & $5.01(0.15)$ & $2.53(0.38)$ & $2.89(0.51)$ & $2.87(0.59)$ & $2.97(0.52)$ \\
Bone mineral density $\left(\mathrm{mg} / \mathrm{cm}^{2}\right)$ & 0 & $396.75(0.39)$ & $465.63(0.15)$ & $483.63(0.38)$ & $508.5(0.52)$ & $555.38(0.59)$ & $559.25(0.52)$ \\
\hline
\end{tabular}

The mean \pm SD of five independent experiments $( \pm \mathrm{SD})$

\section{Statistical analysis}

These data expressed as the mean \pm SD for each group. Statistical difference were analyzed using Scheffe's test. Values of $\mathrm{P}<0.05$ were considered statistically significant.

\section{Histological analysis}

Five days and 1, 2, 4, 6, and 8 weeks after surgery, regenerative bone was extirpated and fixed in $4 \%$ paraformaldehyde phosphate buffer solution. The resected specimens were decalcified in $10 \%$ EDTA (Dojindo, Kumamoto, Japan) for 10 days at $4{ }^{\circ} \mathrm{C}$. The specimens were then dehydrated in a graded series of ethanol, embedded in paraffin, and sectioned transversely at a thickness of $8 \mu \mathrm{m}$. The sections were stained with hematoxylin and eosin. Chondrocyte were identified by alcian bule staining, and osteoclasts were identified by tartrate-resistant acidic phosphate (TRAP) staining using a TRAP/ALP stain kit (Wako, Tokyo, Japan). Histologic examination was performed under a light microscope equipped with a morphometric system connected to a personal computer. 
Takayuki Mashimo et al. Bone Regenerative Process from Periosteum
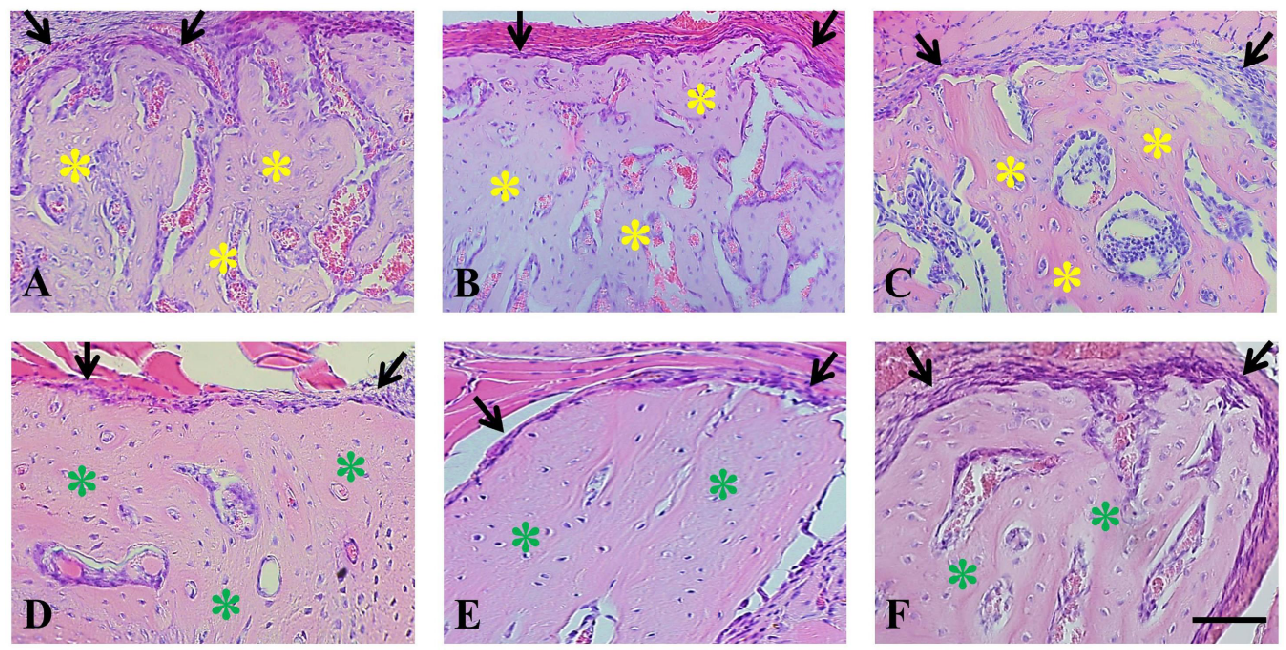

Figure 9. The section at the center of regenerative bone in the PP model (hematoxylin-eosin staining, 200-fold magnification, cross sections, scale bar: $100 \mu \mathrm{m})$

A: 5 days after operation; B: 1 week after operation; C: 2 weeks after operation; D: 4 weeks after operation; E: 6 weeks after operation; F: 8 weeks after operation; (Black arrow: periosteum. Yellow asterisk: woven bone. Green asterisk: lamellar bone.)
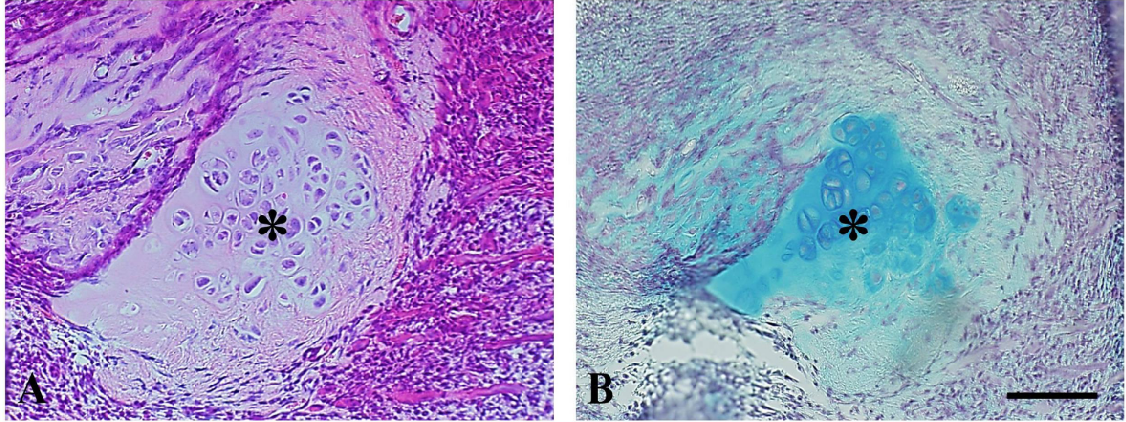

Figure 10. The section at the epiphysis of regenerative bone in the PP model (5 days)

A: hematoxylin-eosin staining, 200-fold magnification, cross sections, scale bar: $100 \mu \mathrm{m}$ (black asterisk: cartilagelike tissue); B: alcian blue staining, 200-fold magnification, cross sections, scale bar: $100 \mu \mathrm{m}$ (black asterisk: cartilage-like tissue)
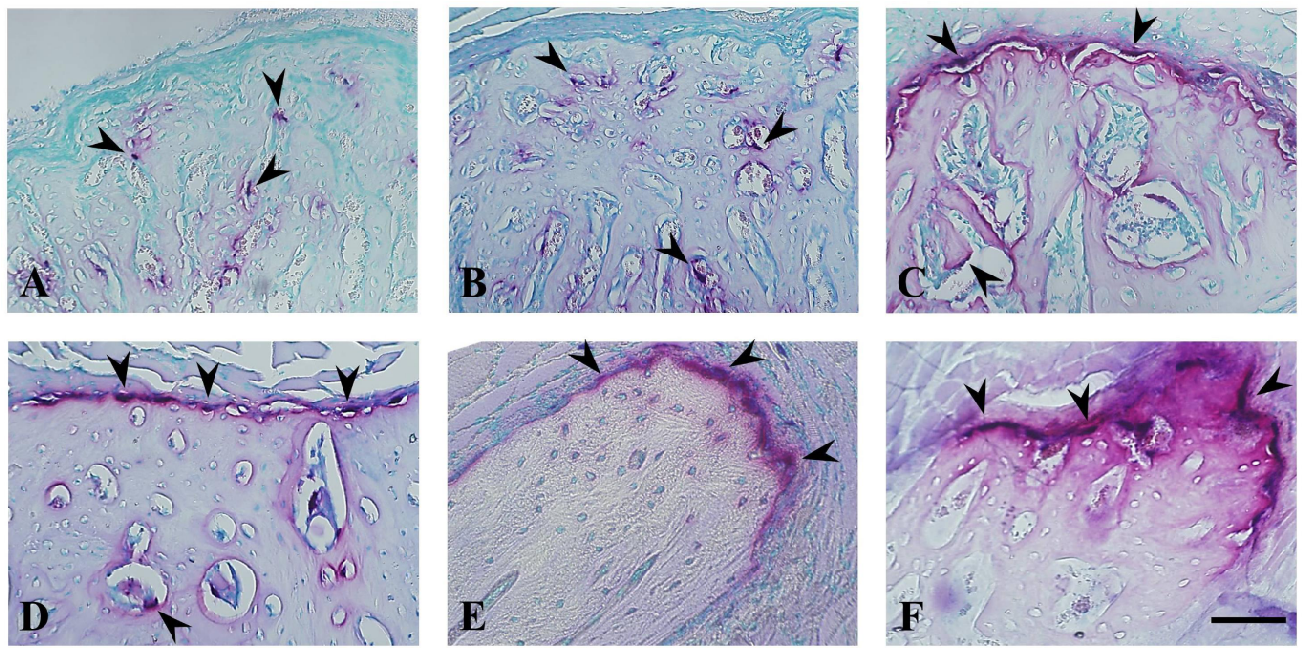

Fig. 11. The section at the center of regenerative bone in the PP model (Tartrate-resistant acidic phosphate (TRAP) staining, 200 -fold magnification, cross sections, scale bar: $100 \mu \mathrm{m}$ ).

A: 5 days after operation; B: 1 week after operation; C: 2 weeks after operation; D: 4 weeks after operation; E: 6 weeks after operation; F: 8 weeks after operation; (Black arrowhead: TRAP ${ }^{+}$osteoclast) 


\section{Results}

\section{Radiographic analysis}

Regenerative bone on the fibula was examined using micro computed tomography and was quantified with reconstruction software (i-VIEW) in cross-sectional and longitudinal planes. Immediately after operation, both groups showed that the fibula had been totally removed (Fig. 3, 4). The CT images of the PP groups showed initial signs of bone formation on the fibula 5 days after operation (Fig. 3-A, B, C; yellow arrow). Bone regeneration began at the center of the diaphysis and gradually progressed to the epiphysis (Fig. 3-A, C). Three-dimensional images of regenerative bone showed that the bone surface was rough at 5 days and 1 week, but then gradually became smooth up to 8 weeks (Fig. 3-C). Morphometric analysis of CT image slices showed that the length of regenerative bone on longitudinal images continued to increase gradually until 8 weeks (Fig. 5, Table 1). The diameter of regenerative bone on cross-sectional images was thickest at 1 week, but then gradually decreased until 8 weeks (Fig. 6, Table 1). At the knee joint, epiphyseal cartilage had increased (Fig. 3-C, yellow arrowhead), but this tissue was not studied because it was not bone regenerated from fibular periosteum, but tissue regenerated from the stump of cartilage. In the PR group, the stump of the fibula at the knee joint increased (Fig. 4-C, yellow arrowhead), but there was not bone regeneration at the center of the diaphysis up to 8 weeks (Fig. 4-C).

\section{Quantitative analysis}

Quantitative analysis of bone regeneration on the fibula was performed with analysis software (3-by-4 viewer 2011) to estimate regenerative bone volume and bone mineral density (Fig. 2-C). In the PP group, regenerative bone volume increased remarkably at 1 week, but decreased at 2 weeks. After 2 weeks the bone volume increased slightly up to 4 weeks, but there was little change thereafter (Fig. 7, Table 1). In contrast, regenerative bone mineral density continued to increase gradually up to 6 weeks. There was little change after 6 weeks (Fig. 7, Table 1). In the PR group, there was no evidence of regenerative bone during the 8 weeks of observation.

\section{Histological findings}

In the PP group, woven bone surrounded by periosteum (Fig. 9-A, black arrow) emerged at the center of regenerative bone at 5 days (Fig. 9-A, yellow asterisks) and was present until 2 weeks (Fig. 9-B, C, yellow asterisks). During 2 to 4 weeks, the woven bone changed to lamellar bone (Fig. 9-D, green asterisks). After 4 weeks, there were no significant changes in the histological findings (Fig. 9-D, E, F). At the epiphysis of regenerative bone, cartilage-like tissue was observed at 5 days (Fig. 10-A, black asterisk). The cartilage-like tissue was stained with alcian blue. In addition, at the diaphysis of that, $\mathrm{TRAP}^{+}$osteoclasts were observed inside the regenerative bone at 5 days and 1 week (Fig. 11-A, B). At 2 weeks and 4 weeks, TRAP $^{+}$osteoclasts were observed not only inside the bone, but also around the bone (Fig. 11-C, D). At 6 weeks and 8 weeks, TRAP $^{+}$osteoclasts were observed only around the bone (Fig. 11-E, F).

\section{Discussion}

The present study showed that when the fibula was totally removed, and the periosteum and its blood supply were preserved (PP group), bone formation was evident. However, when the fibula was removed including the periosteum (PR group), there was no bone formation at all. These results suggest that the periosteum has osteogenic capacity. As mentioned above, many investigators have reported the osteogenic capacity of the periosteum ${ }^{1-18)}$. Bone regeneration from the periosteum was found to depend on mechanical stress and blood supply ${ }^{16,17)}$. However, the most important events in bone formation occur at the cellular level. Recent studies have demonstrated that progenitor cells, such as mesenchymal stem cells (MSCs), exist inside the periosteum, especially in the inner cambium layer ${ }^{31,32)}$. Such MSCs can form bone in vitro and in vivo ${ }^{33,34)}$. MSCs derived from periosteum have a greater osteogenic potential in vitro than those derived from other local tissues, such as adipose tissue or synovium ${ }^{35}$. The PP group showed obvious bone formation, whereas the PR group showed no bone formation. The reason for the difference between the groups was attributed to the presence of MSCs. However, we could not identify the existence of MSCs in periosteum, because we did not investigate marker proteins expressed by MSCs, such as CD29, CD90, and CD105 ${ }^{36}$. Further research is needed on the detection of MSCs by immunohistochemical staining.

In the present study, the time course of bone regeneration was followed in the same subjects. The regenerative bone at defects was examined in detail by means of in vivo micro CT. Micro CT showed that bone formation began at the center of the diaphysis and gradually progressed to the epiphysis in the PP group. Morphometric analysis revealed that the regenerative bone tended to change into long and narrow bone. These changes in the bone appeared to be due to the fibular periosteum promoting extension and growth of the tibia and gastrocnemius muscle. With the use of analysis software, it was possible to quantitatively assess regenerative bone. In the PP group, bone volume clearly increased until 1 week, but then decreased at 2 weeks. After 2 weeks, the bone volume gradually tended to increase again until 8 weeks. However, there was no obvious difference between 4 and 8 weeks. The bone mineral density continued to increase until 6 weeks, although there was no apparent difference between 6 and 8 weeks. Three-dimensional images of regenerative bone showed that the bone surface was rough at 5 days and 1 week, but then gradually became smooth up to 8 weeks. In addition, HE staining of the 
Takayuki Mashimo et al. Bone Regenerative Process from Periosteum

sections showed that regenerative bone from periosteum was initially observed as woven bone at the epiphysis at 5 days. The woven bone gradually changed to lamellar bone up to 8 weeks. There were no significant histological changes from 4 to 8 weeks. These findings showed that immature bone was replaced by mature bone throughout bone remodeling. Remodeling is responsible for changes in bone shape and mass to renew bone, associated with the presence of osteoblasts and osteoclasts ${ }^{37)}$. In this study, we used TRAP staining to detect the localization of osteoclasts. Localization of $\mathrm{TRAP}^{+}$osteoclasts changed with time. TRAP ${ }^{+}$ osteoclasts were found only inside woven bone at 5 days to 1 week, inside and around bone at 2 to 4 weeks, and only around lamellar bone at 6 to 8 weeks. These results suggest that remodeling occurs within regenerative bone at an early stage, and the site of the remodeling shifts to the bone surface with bone mature at the final stage. Remodeling of mature bone is generally thought to occur at the cortical bone surface ${ }^{37}$. Therefore, our study demonstrated the mature process of regenerative bone during 8 weeks. Our findings suggested that the development of mature regenerative bone from periosteum was completed at 4 to 6 weeks. Most previous studies using fibular periosteum examined the repair of segmental bone defects as a model of fracture healing ${ }^{19-21)}$. These studies showed that bone repair involves ossification in the fracture gap and subperiosteal direct bone formation over cortical bone ${ }^{24}$, 25). These previous investigations examined the mechanism of bone fracture healing and did not focus only on the function of the periosteum. Another purpose of these studies of fibular defects was to observe bone regeneration using particulate artificial bone, cells, and biomaterials ${ }^{26,27}$. The results focused on conditions associated with the formation of more regenerative bone. To our knowledge, no previous study used a model lacking fibula in which the blood supply to the periosteum was maintained to directly investigate the periosteal function. The PP model we used specifically allowed assessment of the functions of fibular periosteum for the first time. However, the process of bone regeneration in our model was similar to that previously reported in segmental bone defects. ${ }^{24-27)}$ These regenerative bone in previous reports were thick in the early stage, but gradually became thinner during processes such as fracture healing ${ }^{38)}$. In our previous study, a similar process was observed in a temporomandibular joint defect model in which the periosteum was maintained ${ }^{35)}$. It is interesting to note that the process of bone regeneration from only periosteum was associated with a transient decrease in bone volume. This phenomenon can be explained by the mechanism of bone-fracture healing. After fracture, a hematoma forms beneath the periosteum, and the hematoma is replaced by callus bone, which has a woven structure. The bone undergoes repeated resorption and formation by remodeling and changes to lamellar bone. Consequently, repaired bone becomes thin and functional ${ }^{38)}$. These processes underlie bone regeneration in the PP model.
In addition, nearly all previous studies reported that rat fibular defects were repaired in 4 to 8 weeks ${ }^{24,26,27)}$. We believe that the regenerative process described in past reports followed a similar course as bone regenerated from only periosteum.

Another similarity with previous studies was the ossification pattern of bone regenerated from fibular periosteum. Periosteum of long bones such as the tibia and fibula has osteogenic capacity due to endochondral ossification, while that of membrane bones such as the calvaria has osteogenic capacity due to membranous ossification $^{39)}$. We expected that regenerative bone in this study would be formed by endochondral ossification because our model used fibular periosteum. Endochondral ossification is a process in which bone replaces cartilage. In this type of ossification, formed cartilage is replaced by bone from the central part, and replacement of cartilage by bone then proceeds longitudinally ${ }^{39}$. Radio-opacity images at 5 days indicated where bone replaced cartilage, but could not identify cartilage tissue. However, histological examination at 5 days showed the presence of woven bone at the center of regenerative bone, but cartilage tissue at the epiphysis. This cartilage tissue indicated the top of the epiphysis during endochondral ossification. We thus demonstrated that bone regenerated from fibular periosteum was formed by endochondral ossification.

Clinically, there appear to be two methods to promote the formation of regenerative bone from periosteum. The first method is to induce the formation of regenerative bone by securing a space between bone and periosteum at the donor site. The second method is to form bone at the recipient site by grafting vascularized periosteum. Thus, if bone regenerated from fibular periosteum can be used for bone grafts, it is possible to reconstruct the jawbone without using the fibula itself. We believe this is clinically significant. Our results are promising because they showed that the properties of regenerative bone depend on the observation period. The volume of bone derived from periosteum was greatest at 1 week, but the bone was immature. At 4-8 weeks, the bone was more mature than that at 1 week with respect to bone mineral density and histological characteristics. This study shows that it is necessary to determine the optimal timing of grafting. Future studies are required to investigate whether bone regenerated from fibular periosteum can be used for bone grafts and to define the optimal timing for grafting and the conditions that most efficiently promote the formation of bone regenerated from periosteum.

In conclusion, we successfully followed the process of bone regeneration from fibular periosteum in detail, including the time course of bone regeneration, bone volume, and bone mineral density by means of micro CT. In addition, we demonstrated bone regeneration from only fibular periosteum, unaffected by regeneration from a bone stump. Our results suggested that the peak of bone regeneration from rat fibular periosteum occurred around 4 to 6 weeks. 


\section{Acknowledgements}

This study was supported by Grants-in-Aid for Scientific Research (24593063) from the Japan Society for the Promotion of Science (JSPS), Grants from the Dental Research Center, Sato Funds, Uemura Fund of Nihon University School of Dentistry, as well as special research grants for the development of distinctive education for private schools of Japan.

\section{References}

1. Canalis RF and Burnstein FD. Osteogenesis in vascularized periosteum. Arch Otolaryngol 111: 511-513, 1985

2. Duhamel HL. Observations de physique generale. Histoire de L'Academie Royale des Sciences. De L'imprimerie Royale: 1-3, 1739

3. Mc Williams CA. The function of the periosteum in bone transplants, based on four human transplantations without periosteum, and some animal experiments. Surg Gynec obstet 18: 158-169, 1914

4. Phemister DB. The fate of transplanted bone and regenerative power of its various constitunents. Surg Gynec obstet 19: 303-333, 1914

5. Davis JS and Hunnicutt JA. The osteogenic power of periosteum: with a note on bone transplantation, An experimental study, Ann Surg 61: 672-685, 1915

6. Skoog T. The use of periosteum and Surgicel for bone restoration in congenital clefts of the maxilla: A clinical report and experimental investigation. Scand J Plast Reconstr Surg 1: 113-130, 1967

7. Skoog T. Repair of unilateral cleft lip deformity: Maxilla, nose and lip. Scand J Plast Reconstr Surg 3: 109-133, 1969

8. Ritsila V, Alhopuro S, Gylling U and Rintala A. The use of periosteum for bone formation in congenital clefts of the maxilla: A clinical report. Scand J Plast Reconstr Surg 6: 57-60, 1972

9. King KF. Periosteal pedicle grafting in dogs. J Bone Joint Surg Br 58: 117-121, 1976

10. Finley JM, Acland RD and Wood MB. Revascularized periosteal grafts a new bone without bone grafting. Plast Reconstr Surg 61: 1-6, 1970

11. Buncke HJ, Furnas DW, Gordon L and Achauer BM. Free osteocutaneous flap from a rib to the tibia. Plast Reconstr Surg 59: 799-804, 1977

12. Puckett CL, Hurvitz JS, Metzler MH and Silver D. Bone formation by revascularized periosteal and bone grafts, compared with traditional bone grafts. Plast Reconstr Surg 64: 361-365, 1979

13. Acland RD. Caution about clinical use of vascularized periosteal grafts. Plast Reconstr Surg 62: 290, 1978

14. van den Wilderberg FA, Goris RJ and Tutein NoltheniusPuylaert MB. Free revascularised periosteum transplantation: an experimental study. Br J Plast Surg 37: 226-235, 1984

15. Takato T, Harii K, Nakatsuka T, Ueda K and Ootaka T. Vascularized periosteal grafts: an experimental study using two different forms of tibial periosteum in rabbits. Plast Reconstr Surg 78: 489-497, 1986

16. Takato T, Harii K and Nakatsuka T. Osteogenic capacity of vascularised periosteum: experimental study using rib periosteum in rabbits. Br J Plast Surg 41: 528-532, 1988

17. Uddströmer L and Ritsila V. Osteogenic capacity of periosteal grafts. A qualitative and quantitative study of membranous and tubular bone periosteum in young rabbits, Scand J Plast Reconstr Surg 12: 207-214, 1978

18. Uddströmer L and Ritsila V. Healing of membranous and long bone defects. An experimental study in growing rabbits. Scand J Plast Reconstr Surg 13: 281-287, 1979

19. Oni OO and Gregg PJ. An investigation of the contribution of the extraosseous tissues to the diaphyseal fracture callus using a rabbit tibial fracture model. J Orthop Trauma 5: 480484, 1991

20. Oni OO, Stafford H and Gregg PJ. A study of diaphyseal fracture repair using tissue isolation techniques. Injury 23: 467-470, 1992

21. Li M, Amizuka N, Oda K, Tokunaga K, Ito T, Takeuchi K, Takagi $\mathrm{R}$ and Maeda T. Histochemical evidence of the initial chondrogenesis and osteogenesis in the periosteum of a rib fractured model: implications of osteocyte involvement in periosteal chondrogenesis. Microsc Res Tech 64: 330-342, 2004

22. Taylor GI, Miller GD and Ham FJ. The free vascularized bone graft. A clinical extension of microvascular techniques. Plast Reconstr Surg 55: 533-544, 1975

23. Hidalgo DA. Fibula free flap: a new method of mandible reconstruction. Plast Reconstr Surg 84: 71-79, 1989

24. Chakkalakal DA, Strates BS, Mashoof AA, Garvin KL, Novak JR, Fritz ED, Mollner TJ and McGuire MH. Repair of segmental bone defects in the rat: an experimental model of human fracture healing. Bone 25: 321-332, 1999

25. Kirchen ME, O'Connor KM, Gruber HE, Sweeney JR, Fras IA, Stover SJ, Sarmiento A and Marshall GJ. Effects of microgravity on bone healing in a rat fibular osteotomy model. Clin Orthop Relat Res 318: 231-242, 1995

26. Bluhm AE and Laskin DM. The effect of polyterafluoroethylene cylinders on osteogenesis in rat fibular defects: A preliminary study, J Oral Maxillofac Surg, 53: 163-166, 1995

27. Lazard ZW, Heggeness MH, Hipp JA, Sonnet C, Fuentes AS, Nistal RP, Davis AR, Olabisi RM, West JL and OlmstedDavis EA. Cell-based gene therapy for repair of critical size defects in the rat fibula. J Cellular Bio 112: 1563-1571, 2011

28. Arita K, Saito I and Arai Y. Evaluation of mouse gutter shaped 
Takayuki Mashimo et al. Bone Regenerative Process from Periosteum

$\operatorname{root}(\mathrm{s})$ as a quantitative trait using micro-CT. Pediatr Dent $\mathrm{J}$ 16: 23-27, 2006

29. Osuga N, Yang J, Yamakawa Y, Ninomiya T, Arai Y and Raorao W. Micro-CT observation of rat dental pulp healing after pulpotomy in in vivo study. Pediatr Dent J 16: 132137, 2006

30. Saito T, Mashimo T, Shiratsuchi H, Namaki S, Matsumoto K, Mori Y, Ogasawara T, Arai Y, Honda K and Yonehara Y. Evaluation of regenerative process in a rat model of mandibular condyle defect using in vivo micro $\mathrm{x}$-ray computed tomography. J Hard Tissue Biol 21: 407-414, 2012

31. Choi YS, Noh SE, Lim SM, Lee CW, Kim CS, Im MW, Lee $\mathrm{MH}$ and Kim DI. Multipotency and growth characteristic of periosteum-derived progenitor cells for chondrogenic, osteogenic, and adipogenic differentiation. Biotechnol Lett 30: 593-601, 2008

32. De Bari C, Dell'Accio F, Vanlauwe J, Eyckmans J, Khan IM, Archer CW, Jones EA, McGonagle D, Mitsiadis TA, Pitzalis C and Luyten FP. Mesenchymal multipotency of adult human periosteal cells demonstrated by single-cell lineage analysis. Arthritis Rheum 54: 1209-1221, 2006

33. Nakahara H, Dennis JE, Bruder SP, Haynesworth SE, Lennon
DP and Caplan AI. In vitro differentiation of bone and hypertrophic cartilage from periosteal-derived cells. Exp Cell Res 195: 492-503, 1991

34. Nakahara H, Bruder SP, Goldberg VM and Caplan AI. In vivo osteochondrogenic potential of cultured cells derived from the periosteum. Clin Orthop Relat Res 259: 223-32, 1990

35. Augello A, Kurth TB and De Bari C. Mesenchymal stem cells: a perspective from in vitro cultures to in vivo migration and niches. Eur Cell Mater 20: 121-133, 2010

36. Pittenger MF, Mackay AM and Beck SC. Multilineage potential of adult human mesenchymal stem cells. Science 284: 143-147, 1999

37. Huja SS and Beck FM. Bone remodeling in maxilla, mandible, and femur of young dogs. Anat Rec (Hoboken) 291: 1-5, 2008

38. Rockwood CA and Green DP. Fractures. JB Lippincott: 98100, 1975

39. Fujii T, Ueno T, Kagawa T, Sakata Y and Sugahara T. Comparison of bone formation in grafted periosteum harvested from tibia and calvaria. Microsc Res Tech 69: 580584, 2006 